\title{
Research on Performance Incentive Mechanism of General Practitioners under the Consideration of Reputation Effect
}

\author{
Ma zhi-qiang \\ Jiangsu University \\ Zhu Bin-xin ( $\square$ zhubinxin44@163.com ) \\ Jiangsu University https://orcid.org/0000-0002-8810-7472
}

\section{Research}

Keywords: medical service value, General Practitioners, reputation effect, principal-agent theory, game theory

Posted Date: December 30th, 2019

DOI: https://doi.org/10.21203/rs.2.19728/v1

License: (c) (i) This work is licensed under a Creative Commons Attribution 4.0 International License.

Read Full License 


\section{Research on Performance Incentive Mechanism of General \\ 2 Practitioners under the Consideration of Reputation Effect}

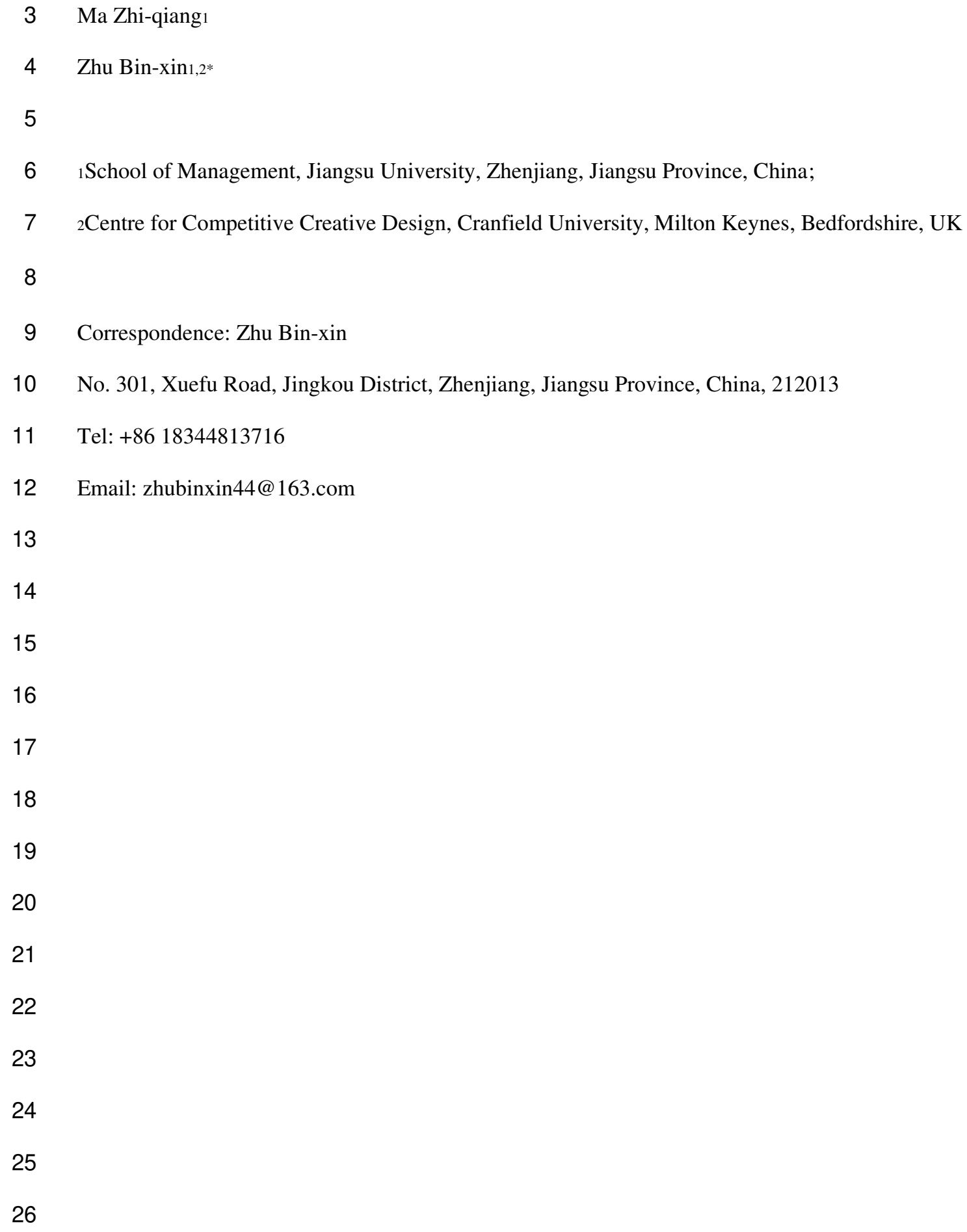




\section{Abstract}

2 Background: In China, General practitioners (GPs) have a weaker reputation than specialists, which leads

3 to their low professional identity. Therefore, GPs' reputation is a bottleneck restricting their participation in

4 skills training and the realization of the service value of community health centre. Because the GPs'

5 reputation accumulation can only rely on the improvement of personal skills, it is necessary to combine the

6 improvement of medical skills to the future economic interests of the GPs, and establishing an effective

7 dynamic reputation incentive mechanism. The purpose of this paper is to explore the dynamic reputation

8 incentive mechanism for GPs, and analyze its performance.

9 Methods: In this paper, a dynamic incentive model of the GPs in two stages under the effect of explicit and

10 implicit reputation is established and solved by using game theory and agent-principal theory.

11 Results: The explicit reputation affects GPs' determined incomes mainly through fixed awards, and the

12 medical effort input in Stage 1 decreases with the performance award in Stage 2 under the reputation

13 mechanism; the increase of the implicit reputation coefficient can improve the medical performance output,

14 the performance award level and the total value of medical service value, and the explicit reputation

15 correction factor plays the opposite role; when the implicit reputation coefficient and explicit reputation

16 correct factor meet certain conditions, the implementation of reputation incentive can lead to the Pareto

17 effect of GPs' medical effort and performance awards.

18 Conclusion: (1) explicit reputation mainly affects GPs' deterministic income in Stage 2 by changing the

19 fixed benefits; (2) the medical efforts in the Stage 1 depend on the unit performance incentive coefficient in

20 both stages, and show a certain ratchet effect; (3) implicit reputation can help to improve the level of 21 medical efforts at each stage; (4) the reputation uncertainty will reduce the level of medical efforts and unit

22 performance incentive at each stage; (5) when the explicit reputation correction coefficient is lower than a

23 certain threshold, the implement of reputation incentive can realize the Pareto effect of medical efforts.

\section{Keywords}

25 medical service value; General Practitioners; reputation effect ; principal-agent theory; game theory 


\section{Background}

2 General practitioners (GPs) are the essential part of community health services, and play an important role

3 in preventive health care, common disease diagnosis and treatment, and patient rehabilitation management

4 at the grassroots level [1]. Previous studies have shown that GPs' training skills can effectively increase the

5 value of community health services. For example, it helps for more active participation in cancer screen,

6 the management improvement of systemic diseases, and better solution to the epidemic public health crisis

$7 \quad[2,3,4,5]$. In China, because the reputation of community health care institutions nowadays is still far lower

8 than the large general hospitals, the patient's trust in the level of community health service technology is

9 relatively low, and the amount of medical visits is not high, which leads to the serious lack of willingness

10 of GPs to invest in medical efforts [6]. According to statistics, the growth rate of primary health care in

11 China from 2014 to 2015 is only $0.9 \%$, which is far lower than $4.3 \%$ of general hospitals [7]. Unlike

12 general hospital doctors, the GPs' reputation accumulation can only rely on the improvement of personal

13 skills and cannot depend on the influence of organizations [8]. It can be seen that combining the

14 improvement of medical skills to the future economic interests of the GPs, and establishing an effective

15 dynamic reputation incentive mechanism are important ways to drive them to participate in skills training,

16 and thus improve the quality of the medical services of community health institutions $[9,10]$.

17 Most studies focus on service value of GPs, but there are little references to research the impact of

18 reputation on medical performance of general practitioners which involves multi-stage objectives and

19 dynamic decision-making [11,12,13,14,15]. Although Jiang and Li, Zhang \& Zhu conducted qualitative

20 studies [9,10], they failed to analyze the internal mechanism and specific operation of reputation incentive

21 mechanism quantitatively. This paper combines the principal-agent theory to explore the dynamic

22 reputation incentive mechanism for GPs on the basis of second-stage game, which provides inspiration for

23 guiding GPs to improve medical efforts and realize the value of community health services.

\section{2. Model Description and Assumption}

26 We considers a two-stage principal-agent mechanism model between community health institutions and

27 GPs. As the agent, GPs pay efforts to improve performance of medical services and gain certain benefits

28 including fixed income and performance rewards from principal community health institutions. 
1 Performance rewards is based on the GPs' medical service performance and adjusted according to the

2 explicit reputation accumulated in the previous stage, which will undoubtedly affect GPs' economic benefit

3 in the future. In addition, GPs also show a certain implicit reputation, that is, the medical costs will be

4 shared at this stage due to the improvement of skills and reputation. The GPs' reputation incentive model is

5 shown in Figure 1.

6

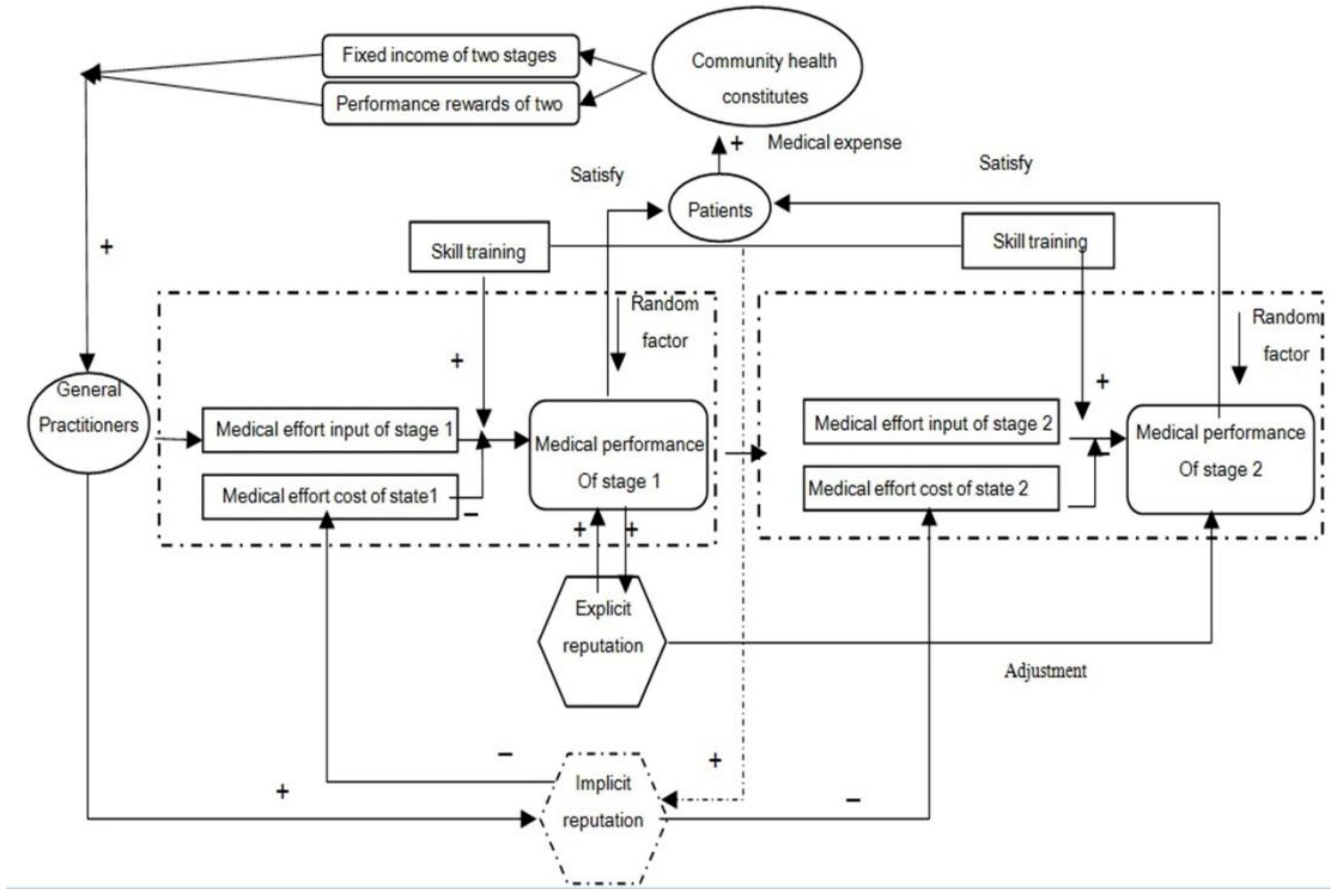

Figure 1 GPs two-stage dynamic reputation incentive mechanism model

GPs medical performance (measured by the quality of the medical services they provided) is determined by their medical efforts, reputation output, and certain random factors, which can be expressed as:

$$
m_{t}=k e_{t}+\lambda r_{t}+\varepsilon_{t}, \quad t=1,2
$$

$k$ is the medical efforts coefficient, which determines the extent to which medical efforts are translated into performance; $e_{t}$ is the degree of medical effort of GPs in the stage $t . \lambda$ is called the reputation output coefficient and $r_{t}$ represents the explicit reputation (inferred as quality ability, qualification or public praise) of GPs in stage $t$. We assume that $r_{t} \sim N\left(a, \sigma_{r}{ }^{2}\right)$ indicating that the GPs' expected reputation is $a$ and exists uncertainty. $\varepsilon_{t}$ represents random factors, and $\varepsilon_{t} \sim N\left(0, \sigma_{\varepsilon}{ }^{2}\right)$. We also assume $\varepsilon_{t}$ and $r_{t}$ are independent. 
1 The information asymmetrical exists between the principal and the agent. That is, at each stage, community

2 health institutions cannot observe the actual medical efforts put by GPs, and only reversely judge it

3 according to the medical performance output. In Stage 1, community health organizations estimate the GP's

4 medical effort to be $e_{1}^{*}$, and observe the actually medical performance $m_{1}$, then revise GPs reputation

5 output to in $\lambda r_{2}$ Stage 2. According to the Shi et al, the revised GPs reputation output $v$ is (Shi, Zhu \&

$6 \quad$ Sheng, 2017):

$$
v=E\left(\lambda r_{2} \mid m_{1}\right)=(1-\tau) \lambda a+\tau\left(m_{1}-k e_{1}^{*}\right)
$$

8 That is to say, the revised reputation is a weighted average of the expected value $\lambda a$ and the actual 9 observed value $m_{1}-k e_{1}^{*}$. The correction coefficient $\tau(0<\tau<1)$ is the proportion of reputation output uncertainty in the total uncertainty of medical performance, namely

$$
\tau=\frac{\lambda^{2} \sigma_{r}{ }^{2}}{\lambda^{2} \sigma_{r}{ }^{2}+\sigma_{\varepsilon}{ }^{2}}
$$

$12 \tau$ indicates the explicit reputation stability. So we can get the variance of the reputation output:

$$
\operatorname{var}(v)=\tau^{2}\left(\lambda^{2} \sigma_{r}^{2}+\sigma_{\varepsilon}^{2}\right)=\lambda^{2} \tau \sigma_{r}^{2}
$$

14 In stage $t$, GPs receive a fixed income $\alpha_{t}$ and unit performance reward $\beta_{t}$. So the total income in stage $t$ is 15 expected to be:

$$
w_{t}=\alpha_{t}+\beta_{t}\left(k e_{t}+\lambda r_{t}+\varepsilon_{t}\right)
$$

17 We assume GPs have risk aversion whose utility function $U=-e^{-\rho \sigma}$, where $\rho$ is the degree of risk 18 aversion, $\omega$ is the real monetary income. Medical cost (such as medical equipment loss, depreciation, 19 energy loss) is a strictly monotonic incremental convex function for GPs' medical efforts, which can be 20 expressed as:

$$
C\left(e_{t}\right)=\frac{u}{2(1+\theta)} e_{t}^{2}
$$

22 Where $1+\theta$ is the implicit reputation coefficient of GPs, that is, due to good reputation, it will result in 23 higher medical service value in the future which is reflected as the cost at present stage. Meanwhile, we 
2 health institutions in stage $t$ is:

$$
U_{t}=\left(1-\beta_{t}\right) m_{t}-\alpha_{t}=\left(1-\beta_{t}\right)\left(k e_{t}+\lambda r_{t}+\varepsilon_{t}\right)-\alpha_{t}
$$

\section{3.Model construction and solution under the reputation incentive mechanism of GPs}

\section{3.1. Stage 2}

7 According to the above description, the GP's expected revenue function in stage 2 is:

$$
w_{2}=\alpha_{2}+\beta_{2}\left(k e_{2}+\lambda r_{2}+\varepsilon_{2}\right)-\frac{u}{2 \varphi} e_{2}^{2}
$$

9 After the reputation is revised, the GP's deterministic revenue can be expressed as:

$$
V_{2}=\alpha_{2}+\beta_{2}\left(k e_{2}+v\right)-\frac{u}{2 \varphi} e_{2}^{2}-\frac{\rho \beta_{2}^{2}}{2}\left(\sigma_{\varepsilon}^{2}+\lambda^{2} \tau \sigma_{r}^{2}\right)
$$

11 Taking into account all the incentive costs, the deterministic net revenue of community health institutions 12 is:

$$
U_{2}=\left(1-\beta_{2}\right)\left(k e_{2}+v\right)-\alpha_{2}
$$

14 Thus, the reputation incentive model of stage 2 can be expressed as followed:

$$
\begin{array}{ll}
\max _{\alpha_{2}, e_{2}, \beta_{2}} & U_{2}=\left(1-\beta_{2}\right)\left(k e_{2}+v\right)-\alpha_{2} \\
\text { s.t. } \quad & (\mathrm{IR}) \alpha_{2}+\beta_{2}\left(k e_{2}+v\right)-\frac{u}{2 \varphi} e_{2}^{2}-\frac{\rho \beta_{2}^{2}}{2}\left(\sigma_{\varepsilon}^{2}+\lambda^{2} \tau \sigma_{r}^{2}\right) \geq F_{2} \\
& (\mathrm{IC}) e_{2} \in \max \left[\alpha_{2}+\beta_{2}\left(k e_{2}+v\right)-\frac{u}{2 \varphi} e_{2}^{2}-\frac{\rho \beta_{2}^{2}}{2}\left(\sigma_{\varepsilon}{ }^{2}+\lambda^{2} \tau \sigma_{r}^{2}\right)\right]
\end{array}
$$

GPs decide the medical effort $e_{2}$ based on individual rationality constraint (IR) and incentive compatibility

17 (IC). $F_{2}$ indicates the retain utility of GPs. According the constraint of IC, the equilibrium $e_{2}^{*}$ is obtained

18 by using the first-order partial derivative method. That is

$$
e_{2}^{*}=\frac{k \varphi \beta_{2}}{u}
$$

20 The principal community health institutions don't not allow GPs to earn benefits higher than $F_{2}$ under rational conditions. Therefore, we can take the equal sign in (IR) of Formula (11). According to Shi et al, 
under the reputation mechanism, $F_{2}$ is the utility that may be generated by the best chance of giving up, i.e.

$2 F_{2}=V_{2}=\eta\left(U_{2}+V_{2}\right)$, where $\eta$ means the bargain power of GPs [15]. So, the deterministic revenue of

3 community health institutions about $\beta_{2}$ can be expressed as

4

$$
U_{2}=(1-\eta)\left(\frac{\varphi k^{2} \beta_{2}}{u}+v-\frac{\varphi k^{2}}{2 u} \beta_{2}^{2}-\frac{\rho\left(\sigma_{\varepsilon}^{2}+\lambda^{2} \tau \sigma_{r}^{2}\right)}{2} \beta_{2}^{2}\right)
$$

Taking the first-order partial derivative of $U_{2}$ to $\beta_{2}$, the optimal performance incentive coefficient is $\beta_{2}{ }^{*}$ obtained. Then we put it in Formula (12) and get the equilibrium medical effort $e_{2}^{*}$ :

$$
\beta_{2}^{*}=\frac{\varphi k^{2}}{\varphi k^{2}+u \rho\left({\sigma_{\varepsilon}}^{2}+\lambda^{2} \tau \sigma_{r}^{2}\right)}, e_{2}^{*}=\frac{\varphi^{2} k^{3}}{u\left(\varphi k^{2}+u \rho\left({\sigma_{\varepsilon}}^{2}+\lambda^{2} \tau \sigma_{r}^{2}\right)\right)}
$$

And the fixed income is:

$$
\alpha_{2}=\frac{u \rho\left(\sigma_{\varepsilon}^{2}+\lambda^{2} \tau \sigma_{r}^{2}\right)}{\varphi k^{2}+u \rho\left(\sigma_{\varepsilon}^{2}+\lambda^{2} \tau \sigma_{r}^{2}\right)}\left(\frac{\varphi^{2} k^{4}}{u\left(\varphi k^{2}+u \rho\left(\sigma_{\varepsilon}^{2}+\lambda^{2} \tau \sigma_{r}^{2}\right)\right)}+v\right)-(1-\eta)\left(\frac{(1+\theta)^{2} k^{4}}{2 u\left(\varphi k^{2}+u \rho\left(\sigma_{\varepsilon}^{2}+\lambda^{2} \tau \sigma_{r}^{2}\right)\right)}+v\right)
$$

Hence, the economic benefit of GPs and the community health institutions, and the total value of the medical services (the sum of them two) in stage 2 are:

$$
\begin{aligned}
& U_{2}=(1-\eta)\left(\frac{\varphi^{2} k^{4}}{2 u\left(\varphi k^{2}+u \rho\left(\sigma_{\varepsilon}^{2}+\lambda^{2} \tau \sigma_{r}^{2}\right)\right)}+v\right), V_{2}=\eta\left(\frac{\varphi^{2} k^{4}}{2 u\left((1+\theta) k^{2}+u \rho\left(\sigma_{\varepsilon}^{2}+\lambda^{2} \tau \sigma_{r}^{2}\right)\right)}+v\right) \\
& M V_{2}=U_{2}+V_{2}=\frac{\varphi^{2} k^{4}}{2 u\left(\varphi k^{2}+u \rho\left(\sigma_{\varepsilon}^{2}+\lambda^{2} \tau \sigma_{r}^{2}\right)\right)}+v
\end{aligned}
$$

\subsection{Stage 1}

Let us go back to stage 1 . Under the reputation mechanism, both parties would aim at maximizing the sum of two stages deterministic revenues in Stage 1. Since Stage 1 is the initial phase, it is not possible to correct GP's dominant reputation. Therefore, the decision goal of GPs is:

$$
V=\left(\alpha_{1}+\beta_{1}\left(k e_{1}+\lambda a\right)-\frac{u}{2 \varphi} e_{1}^{2}-\frac{\rho \beta_{1}^{2}}{2}\left(\sigma_{\varepsilon}^{2}+\lambda^{2} \sigma_{r}^{2}\right)\right)+\left(\alpha_{2}+\beta_{2}\left(k e_{2}+v\right)-\frac{u}{2 \varphi} e_{2}^{2}-\frac{\rho \beta_{2}^{2}}{2}\left(\sigma_{\varepsilon}^{2}+\lambda^{2} \tau \sigma_{r}^{2}\right)\right)
$$

The decision goal of community health institutions is:

$$
U=U_{1}+U_{2}=\left(1-\beta_{1}\right)\left(k e_{1}+\lambda a\right)-\alpha_{1}+\left(1-\beta_{2}\right)\left(k e_{2}+v\right)-\alpha_{2}
$$

Thus, the reputation incentive model of Stage 1 can be expressed as followed: 


$$
\max _{\alpha_{1}, e_{1}, \beta_{1}} U=\left(1-\beta_{1}\right)\left(k e_{1}+\lambda a\right)-\alpha_{1}+\left(1-\beta_{2}\right)\left(k e_{2}+v\right)-\alpha_{2}
$$

$$
\begin{array}{ll}
\text { s.t. } & (\mathrm{IR})\left(\alpha_{1}+\beta_{1}\left(k e_{1}+\lambda a\right)-\frac{u}{2 \varphi} e_{1}^{2}-\frac{\rho \beta_{1}^{2}}{2}\left(\sigma_{\varepsilon}^{2}+\lambda^{2} \sigma_{r}^{2}\right)\right)+\left(\alpha_{2}+\beta_{2}\left(k e_{2}+v\right)-\frac{u}{2 \varphi} e_{2}^{2}-\frac{\rho \beta_{2}^{2}}{2}\left(\sigma_{\varepsilon}^{2}+\lambda^{2} \tau \sigma_{r}^{2}\right)\right) \geq F \\
& \text { (IC) } e_{i 1} \in \max \left[\left(\alpha_{1}+\beta_{1}\left(k e_{1}+\lambda a\right)-\frac{u}{2 \varphi} e_{1}^{2}-\frac{\rho \beta_{1}^{2}}{2}\left(\sigma_{\varepsilon}^{2}+\lambda^{2} \sigma_{r}^{2}\right)\right)+\left(\alpha_{2}+\beta_{2}\left(k e_{2}+v\right)-\frac{u}{2 \varphi} e_{2}^{2}-\frac{\rho \beta_{2}^{2}}{2}\left(\sigma_{\varepsilon}^{2}+\lambda^{2} \tau \sigma_{r}^{2}\right)\right)\right]
\end{array}
$$

2 Here, $F$, the sum of the two-stage retention utility is an exogenous constant. Under the reputation mechanism, the performance of Stage 1 would change the reputation output $v$ of Stage 2 , hence affect the level of medical efforts in Stage 2. Substituting $v=(1-\tau) \lambda a+\tau\left(m_{1}-k e_{1}^{*}\right)$ into (IC) condition of Formula (19), and taking the first-order partial derivative $U_{2}$ of to $e_{1}$, we can get

$$
e_{1}^{*}=\frac{\varphi k\left(\beta_{1}-\tau \beta_{2}\right)}{u}
$$

$$
U=\left(k e_{1}\left(\beta_{1}\right)+\lambda a-\frac{u}{2 \varphi} e_{1}\left(\beta_{1}\right)^{2}-\frac{\rho \beta_{1}^{2}}{2}\left(\sigma_{\varepsilon}^{2}+\lambda^{2} \sigma_{r}^{2}\right)\right)+\left(k e_{2}+(1-\tau) \lambda a+\tau\left(m_{1}-k e_{1}\left(\beta_{1}\right)\right)-\frac{u}{2 \varphi} e_{2}^{2}-\frac{\rho \beta_{2}^{2}}{2}\left(\sigma_{\varepsilon}^{2}+\lambda^{2} \tau \sigma_{r}^{2}\right)\right)-F
$$

9 The equilibrium performance incentive coefficient $\beta_{1}^{*}$ and medical effort $e_{1}^{*}$ are obtained by the firstorder partial derivative method:

$$
\begin{aligned}
& \beta_{1}^{*}=\frac{\varphi k^{2}\left(1-\tau+\tau \beta_{2}^{*}\right)}{\varphi k^{2}+u \rho\left(\sigma_{\varepsilon}{ }^{2}+\lambda^{2} \sigma_{r}^{2}\right)}=\frac{\varphi k^{2}\left(\varphi k^{2}+u \rho\left(\sigma_{\varepsilon}{ }^{2}+\lambda^{2} \tau \sigma_{r}{ }^{2}\right)(1-\tau)\right)}{\left(\varphi k^{2}+u \rho\left(\sigma_{\varepsilon}{ }^{2}+\lambda^{2} \sigma_{r}{ }^{2}\right)\right)\left(\varphi k^{2}+u \rho\left(\sigma_{\varepsilon}{ }^{2}+\lambda^{2} \tau \sigma_{r}^{2}\right)\right)}, \\
& e_{1}^{*}=\frac{k \varphi\left(\varphi k^{2}(1-\tau)-u \rho \sigma_{\varepsilon}{ }^{2} \tau \beta_{2}^{*}\right)}{u\left(\varphi k^{2}+u \rho\left(\sigma_{\varepsilon}{ }^{2}+\lambda^{2} \sigma_{r}{ }^{2}\right)\right)}=\frac{\varphi^{2} k^{3}\left(\varphi k^{2}(1-\tau)+u \rho\left((1-2 \tau) \sigma_{\varepsilon}{ }^{2}-\lambda^{2} \tau^{2} \sigma_{r}{ }^{2}\right)\right)}{u\left(\varphi k^{2}+u \rho\left(\sigma_{\varepsilon}{ }^{2}+\lambda^{2} \sigma_{r}{ }^{2}\right)\right)\left(\varphi k^{2}+u \rho\left(\sigma_{\varepsilon}{ }^{2}+\lambda^{2} \tau \sigma_{r}{ }^{2}\right)\right)}
\end{aligned}
$$

The deterministic revenue of community health institutions and total value of medical services are:

13

$$
\begin{aligned}
& U_{1}^{*}=\left(\left(k e_{1}^{*}+\lambda a\right)-\frac{u}{2 \varphi} e_{1}^{* 2}-\frac{\rho \beta_{1}^{* 2}\left(\sigma_{\varepsilon}^{2}+\lambda^{2} \sigma_{r}^{2}\right)}{2}\right)+\eta\left(\frac{\varphi^{2} k^{4}}{2 u\left(\varphi k^{2}+u \rho\left(\sigma_{\varepsilon}^{2}+\lambda^{2} \tau \sigma_{r}^{2}\right)\right)}+v\right)-F \\
& M V_{1}^{*}=U_{1}^{*}+V_{1}^{*}=\left(k e_{1}^{*}+\lambda a\right)-\frac{u}{2 \varphi} e_{1}^{* 2}-\frac{\rho \beta_{1}^{* 2}\left(\sigma_{\varepsilon}^{2}+\lambda^{2} \sigma_{r}^{2}\right)}{2}=\frac{(1+\tau) \varphi k^{2}\left(\beta_{1}^{*}-\tau \beta_{2}^{*}\right)}{2 u}-\frac{\rho \tau \beta_{1}^{*} \beta_{2}^{*}}{2}\left(\sigma_{\varepsilon}^{2}+\lambda^{2} \sigma_{r}^{2}\right)
\end{aligned}
$$

14

\section{Incentive mechanism without consideration of reputation}


1 In this case, GPs' medical performance has nothing to do with reputational factors such as qualifications

2 and public praise. Therefore, the incentive mechanism of Stage 1 is consistent with that of Stage 2 . The

3 medical performance can be expressed as:

$$
m=k e+\xi
$$

5 Where $\xi$ is the uncertainty of medical performance caused by factors such as medical policy and medical

6 technology advancement, and we can assume that $\xi \sim N\left(0, \lambda^{2} \sigma_{r}{ }^{2}+\sigma_{\varepsilon}{ }^{2}\right)$. Without considering the implicit

7 reputation, the medical effort cost will be changed into $\frac{u}{2} e^{2}$ and the parameters of fixed income and unit 8 performance reward are unchanged. Therefore, the economic benefit is $9 \quad V=\alpha+\beta k e-\frac{u}{2} e^{2}-\frac{\rho \beta^{2}}{2}\left(\sigma_{\varepsilon}{ }^{2}+\lambda^{2} \sigma_{r}^{2}\right)$. The incentive model is expressed as follows:

$$
\begin{array}{ll}
\max _{\alpha_{2}, e_{i 2}, \beta_{i 2}} & U=(1-\beta) k e-\alpha \\
\text { s.t. } \quad & (\mathrm{IR}) \alpha+\beta k e-\frac{u}{2} e^{2}-\frac{\rho \beta^{2}}{2}\left(\sigma_{\varepsilon}{ }^{2}+\lambda^{2} \sigma_{r}{ }^{2}\right) \geq F_{0} \\
& (\mathrm{IC}) e \in \max \left[\alpha+\beta k e-\frac{u}{2} e^{2}-\frac{\rho \beta^{2}}{2}\left(\sigma_{\varepsilon}{ }^{2}+\lambda^{2} \sigma_{r}{ }^{2}\right)\right]
\end{array}
$$

11 The expressions for equilibrium medical effort and performance incentive coefficient are:

$$
\beta^{*}=\frac{k^{2}}{k^{2}+u \rho\left({\sigma_{\varepsilon}}^{2}+\lambda^{2} \sigma_{r}^{2}\right)}, e^{*}=\frac{k^{3}}{u\left(k^{2}+u \rho\left(\sigma_{\varepsilon}^{2}+\lambda^{2} \sigma_{r}^{2}\right)\right)}
$$

\section{5. Results}

\section{$14 \quad 5.1$ Sensitivity analysis results}

15 Conclusion 1:(1) $\beta_{2}^{*}$ and $v$ are irrelevant; (2) when $\eta>\beta_{2}^{*}=\frac{\varphi k^{2}}{\varphi k^{2}+u \rho\left(\sigma_{\varepsilon}{ }^{2}+\lambda^{2} \tau \sigma_{r}{ }^{2}\right)}$, reputation output

16 has a positive effect on fixed income $\alpha_{2}$; when $\eta<\beta_{2}{ }^{*}$, reputation output has a negative effect on $\alpha_{2}$.

17 Conclusion 1 indicates that the GPs' explicit reputation mechanism affects the future economic benefit by

18 adjusting their fixed income,. In other words, in Stage 2, community health institutions should change the

19 GPs' fixed income based on the reputation $m_{1}$ gained from the observed previous medical performance.

20 When the bargain ability is high $\left(\eta>\beta_{2}{ }^{*}\right)$, due to the higher medical efforts in stage 1, the explicit 
1 reputation output has a positive effect on fixed benefits. Vice versa. Therefore, from the perspective of

2 reputation revenue, GPs should actively improve professional skills to enhance the initial stage's medical

3 performance and their bargaining power, so as to obtain higher fixed income in the future.

4 Conclusion 2: (1) $e_{1}^{*}$ is positively related to $\beta_{1}^{*}$, negatively related to $\beta_{2}^{*} ;(2) e_{2}^{*}$ is only positively related 5 with $\beta_{2}{ }^{*}$, while has no relationship with $\beta_{1}{ }^{*}$.

6 This conclusion indicates that, in final stage (Stage 2) the reputation mechanism does not work. Therefore, 7 the medical efforts are only related to the performance incentive coefficient. However, medical efforts of 8 Stage 1 is related to two stages' performance incentive coefficient, and will decrease as the performance 9 incentive coefficient of Stage 2 increases. That is, reputation will have a certain ratchet effect. We can learn

10 from $\frac{\partial e_{1}^{*}}{\partial \beta_{2}}=\frac{-\varphi k \tau}{u}$ that, the ratchet effect is positively correlated with the medical effort output coefficient $k$, 11 and the reputation correction coefficient $\tau$, negatively correlated with effort cost coefficient $u$. Therefore, it 12 is unfavorable of too much use of reputation to modify GPs' performance outcomes.

13 Conclusion 3: (1) $e_{2}^{*}$ is positively related to $\varphi, \beta_{2}{ }^{*}$ is positively related to $\varphi ;(2) e_{1}^{*}$ is positively related to $14 \varphi, \beta_{1}^{*}$ is positively related to $\varphi$.

15 This conclusion suggests that the effect of implicit reputation has a continuously positive effect on GPs' 16 efforts. No matter at which stage, the higher the implicit reputation, the greater the level of unit 17 performance rewards given by the community health organization, so the higher input level of GPs medical 18 efforts. Therefore, continuous participation in cost-reducing training, improvement of medical proficiency, 19 provision of efficient services for patients and continuous improvement of implicit reputation are conducive 20 to enhancing the effect of reputation incentive.

21 Conclusion 4: (1) $e_{1}^{*}$ is negatively related to $\tau, \beta_{1}^{*}$ is negatively related to $\tau ;(2) e_{2}^{*}$ is negatively related to $22 \tau, \beta_{2}^{*}$ is negatively related to $\tau$.

23 This conclusion shows that the reputation correction coefficient always plays a negative role in the effect of 24 reputation incentives. In Stage 1, the higher the reputation correction coefficient, the more uncertain the 25 patients are about the GP's ability, the cumulative reputation has a more pronounced impact on future 
1 medical performance, the ratchet effect is enhanced, and the GP's medical efforts are reduced. In Stage 2,

2 although there is no need to consider future revenues, the improvement of the reputation correction

3 coefficient will increase the uncertainty of the medical performance output at this stage and they will avoid

4 revenue risk by reducing medical efforts.

5 Conclusion 5: (1) $M V_{2}$ is positively related to $v$, negatively related to $\tau$, and positively related to $\varphi$; (2)

$6 M V_{1}$ is negatively related to $\tau$, positively related to $\varphi$, and uncorrelated with $v$.

7 We can see that, the higher the revised GP's reputation performance output $v$, the better the value of future 8 medical services; meanwhile, the current service value will not be affected. In addition, the uncertainty of

9 explicit reputation $\tau$ will reduce the total service value too, which means GPs should also strive to 10 maintain the stability of their reputation. Finally, the total value of medical services will increase as the 11 implicit reputation coefficient $\varphi$ increases. Therefore, cost-reduction training must be introduced to GP, 12 which will help to enhance GP's implicit reputation and contribute to the realization of the total value of 13 community health institutions.

14

\section{$15 \quad$ 5.2. Comparative analysis and simulation results}

16 Conclusion 6: $e^{*}<e_{2}^{*}, \beta^{*}<\beta_{2}^{*}$.

17 The conclusion shows that GPs' medical efforts and performance incentive coefficient in stage 2 are 18 improved under the reputation incentive mechanism. On the one hand, as the effect of implicit reputation is 19 continuous, the effort cost is higher than that under the non-reputation mechanism; on the other hand, the 20 variance of the medical performance output revised by the explicit reputation is $\lambda^{2} \tau \sigma_{r}^{2}+\sigma_{\varepsilon}^{2}$, lower than $21 \lambda^{2} \sigma_{r}^{2}+\sigma_{\varepsilon}^{2}$ when there is no reputation incentive. That is, the medical performance uncertainty in Stage 2 22 will be reduced, which will drive GPs to pay higher efforts and obtain greater medical performance.

24 Conclusion 7:(1) when $\varphi k^{2}\left((\varphi-1)\left(\sigma_{\varepsilon}^{2}+\lambda^{2} \sigma_{r}^{2}\right)-\tau\left(\sigma_{\varepsilon}^{2}+\lambda^{2} \tau \sigma_{r}^{2}\right)\right)>(\varphi \tau+1-\varphi) u \rho\left(\sigma_{\varepsilon}^{2}+\lambda^{2} \sigma_{r}^{2}\right)\left(\sigma_{\varepsilon}^{2}+\lambda^{2} \tau \sigma_{r}^{2}\right) \quad$ is 25 satisfied, we have $\beta^{*}<\beta_{1}^{*} ; \quad$ otherwise, $\quad \beta^{*}>\beta_{1}^{*} ; \quad$ (2) when 
$1 \varphi^{2}\left(\varphi k^{2}(1-\tau)+u \rho\left((1-\tau) \sigma_{\varepsilon}^{2}-\tau\left(\sigma_{\varepsilon}^{2}+\lambda^{2} \tau \sigma_{r}^{2}\right)\right)\right)\left(k^{2}+u \rho\left(\sigma_{\varepsilon}^{2}+\lambda^{2} \sigma_{r}^{2}\right)\right)>\left(\varphi k^{2}+u \rho\left(\sigma_{\varepsilon}^{2}+\lambda^{2} \sigma_{r}^{2}\right)\right)\left(\varphi k^{2}+u \rho\left(\sigma_{\varepsilon}^{2}+\lambda^{2} \tau \sigma_{r}^{2}\right)\right)$ is satisfied, we

2 have $e^{*}<e_{1}^{*} ;$ otherwise, $e^{*}>e_{1}^{*}$.

3 The conclusion shows that only when the explicit reputation correction coefficient $\tau$ and the implicit

4 reputation coefficient $\varphi$ satisfy certain conditions, the reputation incentive mechanism will be beneficial to

5 simultaneously improve GPs' medical efforts and performance incentive coefficient in Stage 1. For the

6 complex relationship between medical efforts coefficient and performance incentive coefficient, the

7 simulation is carried out below. Let us set medical effort conversion coefficient $k=70$, explicit reputation

8 output coefficient $\lambda=1$, random variance $\sigma_{\varepsilon}{ }^{2}=80$, medical effort cost coefficient $u=50$, GPs' risk

9 aversion coefficient $\rho=2$. Boundary curves of $\beta_{1}^{*}=\beta^{*}$ and $e_{1}^{*}=e^{*}$ with respect to $\tau$ and $\varphi$ are

10 respectively made, then region maps of $\beta_{1}^{*}>\beta^{*}, e_{1}^{*}>e^{*}$ are obtained (Figure 2 and Figure 3).

11

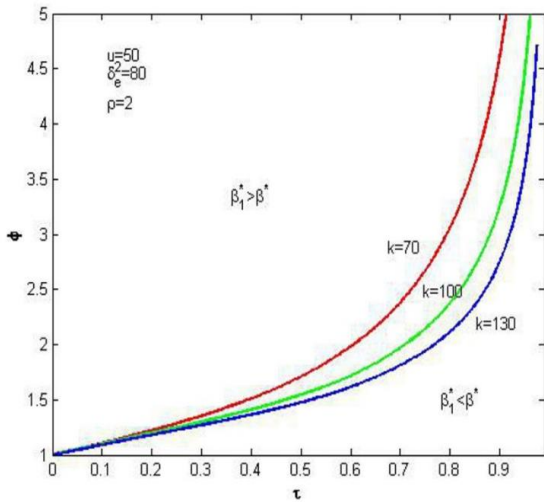

Figure 2 The effect of $\varphi, \tau$ on relationship

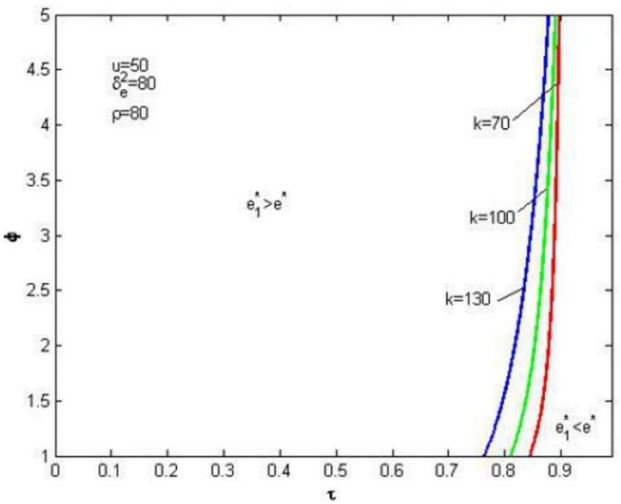

Figure 3 the effect of $\varphi, \tau$ on relationship

of $\beta_{1}^{*}$ and $\beta^{*}$

$$
\text { of } e_{1}^{*} \text { and } e^{*} \text {. }
$$

We can see clearly that the area at the upper left of the curve in Figure 2 satisfies $\beta_{1}^{*}>\beta^{*}$, that is, within the parameter range of the region, the unit performance incentive coefficient of both stage is higher than under the non-reputation mechanism, and the reputation incentive achieves the Pareto effect of performance encouragement. This case can appear if the implicit reputation coefficient $\varphi$ is higher or the explicit reputation correction coefficient $\tau$ is lower than a certain threshold. In addition, it can also be seen that the larger the $k$, the lower the position of the boundary curve, explaining that the greater performance 
conversion of medical efforts causes the higher probability of Pareto effect of performance encouragement,

2 and the greater possibility that GPs will receive performance benefit from reputation incentives.

3 Figure 3 shows that the slope of the boundary curve of $e_{1}^{*}=e^{*}$ is very large, almost perpendicular to the

4 horizontal axis and the left region of it simultaneously satisfy $e_{1}^{*}>e^{*}$ and $e_{2}^{*}>e^{*}$. That is, the reputation

5 incentive can lead to the Pareto effect of medical efforts. We can see that $\tau$ has a significant impact on

6 GP's medical efforts. When $\tau$ is lower than a threshold, a nearly small $\varphi$ can achieve the Pareto effect;

7 while greater than this threshold, even if $\varphi$ is high, it is difficult to achieve. In addition, the larger the $k$,

8 the more the boundary curve shifts to the left, and the smaller the region of $e_{1}^{*}>e^{*}$. This shows that the

9 performance conversion of medical efforts has less influence on GPs' medical efforts than the non-

10 reputation incentive. The more easily medical efforts are translated into performance, the more the

11 community health organizations need to rely on stable reputation output to motivate GPs.

\section{6.Conclusions and Implications}

\section{$14 \quad 6.1$ Conclusions}

15 The medical performance of GPs plays an important role in realizing the service value of 16 community health system. GPs with good reputation have obviously advantages in attracting 17 patients and reducing medical risks. In this paper, principal-agent model is used to study the GPs' 18 reputation incentive mechanism. Considering that community health institutions adjust the 19 explicit reputation according to the performance of GPs in Stage 1, the expected medical 20 performance in Stage 2 is changed. We define implicit reputation as the sharing of effort cost due 21 to the improvement of skills and reputation.

22 The main conclusions of this study are as follows: (1) explicit reputation mainly affects GPs' 23 deterministic income in Stage 2 by changing the fixed benefits; (2) the medical efforts in the

24 Stage 1 depend on the unit performance incentive coefficient in both stages, and show a certain 25 ratchet effect; (3) implicit reputation can help to improve the level of medical efforts at each stage, 26 which will improve the economic benefits of community health institutions and the whole 
medical service value of health system; (4) the reputation uncertainty will reduce the level of

2 medical efforts and unit performance incentive at each stage, and is not conducive to the service

3 value of medical system; (5) when the explicit reputation correction coefficient is lower than a

4 certain threshold, the implement of reputation incentive can realize the Pareto effect of medical

5 efforts in each stage, and the performance conversion rate of medical effort can help to reduce the

6 threshold.

\section{$7 \quad 6.2$ Implications}

8 The conclusions provide useful implications to improve the GPs' medical performance in 9 community health constitutions. It is necessary for community health constitutions to establish a

10 long-term reputation incentive mechanism and improve the dynamic reputation evaluation system

11 for GPs. In view of the complexity of GPs' reputation evaluation, it is necessary to refine the

12 evaluation indicators and consider such factors as the outpatient workload, the number and

13 composition of residents, the quality of service and patient satisfaction, so as to effectively reflect

14 GPs' comprehensive ability formed in the process of long-term dynamic medical service.

15 Furthermore, reputation evaluation cannot be done once for all, but the reputation score should be

16 constantly adjusted according to the pre-performance of GPs, as the basis for the post-

17 performance reward. They can also make full use of the current network and other media means

18 to attract more people to participate in reputation evaluation, and establish a social evaluation and

19 supervision mechanism of professional reputation.

20 The standardized GPs' skills training system should be established so as to effectively improve

21 their medical quality and skill level. In view of the different roles of explicit reputation and

22 implicit reputation, the categories of medical training should be classified as skill-enhancing,

23 quality-improving and cost-reducing. In this way, we can increase the service quality and

24 marginal medical performance. Meanwhile, improving the proficiency of GPs' daily medical

25 service process in order to reduce the cost of the medical effort and increase the implicit

26 reputation coefficient. What's more, GPs should establish the idea of lifelong learning and 
1 training, divide their career into different stages, and set different training contents and

2 curriculum plans respectively.

3 Community health institutions must devote themselves to improving the internal management

4 mechanism and strengthening patients' trust in GPs, which will reduce the negative impact of

5 explicit reputation uncertainty coefficient . Firstly, perfect community medical information

6 disclosure system is very important to reduce information asymmetry between GPs and patients.

7 That is, community health institutions should regularly publish detailed information on medical

8 services, medicines and GPs' qualifications and abilities. This is not only conducive to balance

9 the distribution of medical information between the supply and demand side, but also indirectly

10 form an urge mechanism for GPs to keep learningand reduce the uncertainty of GPs' reputation.

11 Secondly, community health institutions should not only focus on the personal reputation of GPs,

12 but also on the expansion of the reputation of the whole community health care system from

13 internal management mechanism of themselves.

14

15

16

17

18

19

20 、

21

22 
List of abbreviations

\begin{tabular}{|c|c|}
\hline Abbreviations & Description \\
\hline$m_{t}$ & GPs' medical performance of stage $t$ \\
\hline$e_{t}$ & GPs' medical effort of in the stage $t$ \\
\hline$r t$ & GPs' explicit reputation of stage $t$, and $r_{t} \sim N\left(a, \sigma_{r}^{2}\right)$ \\
\hline$\sigma_{r}$ & Standard deviation of $r_{t}$ \\
\hline$\varepsilon_{t}$ & $\begin{array}{l}\text { Random factors of medical performance of stage } t \text {, and } \\
\varepsilon_{t} \sim N\left(0, \sigma_{\varepsilon}^{2}\right)\end{array}$ \\
\hline$\sigma_{\varepsilon}$ & Standard deviation of $\varepsilon_{t}$ \\
\hline$k$ & $\begin{array}{l}\text { Medical efforts coefficient, which determines the extent medical } \\
\text { efforts are translated into performance }\end{array}$ \\
\hline$\lambda$ & $\begin{array}{l}\text { The reputation output coefficient, which determines the extent } \\
\text { explicit reputation are translated into performance }\end{array}$ \\
\hline$v$ & The revised GPs reputation output \\
\hline$\tau$ & $\begin{array}{l}\text { The correction coefficient . that is , the proportion of reputation } \\
\text { output uncertainty in the total uncertainty of medical performance }\end{array}$ \\
\hline$\alpha_{t}$ & GPs' fixed income of stage $t$ \\
\hline$\beta_{t}$ & GPs' unit performance reward of stage $t$ \\
\hline$w_{t}$ & GPs' total benefit of stage $t$ \\
\hline$\rho$ & Degree of GPs' risk aversion \\
\hline$C\left(e_{t}\right)$ & GPs' total medical cost of stage $t$ related to $e_{t}$ \\
\hline$u$ & GPs' medical effort cost coefficient \\
\hline$\varphi$ & The implicit reputation coefficient of GPs. \\
\hline$U_{t}$ & $\begin{array}{l}\text { The total income of the principal community health institutions } \\
\text { (Principal) of stage } t\end{array}$ \\
\hline$V_{t}$ & GPs' total deterministic revenue of stage $t$ \\
\hline$F_{t}$ & GPs' retain utility of stage $t$ \\
\hline$\eta$ & Bargain power of GPs \\
\hline$\xi$ & $\begin{array}{l}\text { The uncertainty of GPs' medical performance without } \\
\text { consideration of reputation }\end{array}$ \\
\hline
\end{tabular}


2 Declarations

3 Ethics approval and consent to participate

$4 \quad$ Not applicable

5

6 Consent for publication

7 I Binxin Zhu give my consent for information about myself to be published in Archives

8 of Public Health.

9

10 Availability of data and material

11 The data and material that support the findings of this study are available from the

12 corresponding author [Binxin Zhu], upon reasonable request.

13

14 Competing interests

15 The authors declare that they have no competing interests.

16

17 Funding

18 This work was supported by the National Nature Science Foundation of China (NSFC)

19 [grant number 71573109].

20

21 Authors' contributions

22 Professor Zhiqiang Ma constructed the models; Binxin Zhu conceived and designed the

23 analysis, performed the analysis and wrote the paper.

24 


\section{Acknowledgements}

2 Not applicable

3

4

\section{References}

[1] Agustin, C., \& Singh, J. (2005). Curvilinear effects of consumer loyalty determinants in relational exchanges. Journal of Marketing Research, 42, 96-108.

[2] Zhou, S. L.(2001). Study on general practitioner's service strategy. General Practitioners, 10(2), $55-56$.

[3] Thu, T. E., Charles, R., \&Froger, P.(2013). The impact of a General Practitioner Training Programme on the Colorectal Cancer Screening Participation Rate. Sante Publique. 25(6), 775-783.

[4] Ellis, J., Rafi, I., \&Smith, H.(2013). Identifying current training provision and future training needs in allergy available for UK general practice trainees National cross-sectional survey of general practitioner specialist training programme directors. Primary Care Respiratory Journal of the General Practice Airways Group, 22(1), 19-22.

[5] Kunin, M., Engelhard, D., \&Piterman, L.(2013). Response of general practitioners to infectious disease public health crises: An integrative systematic review of the literature. Disaster Medicine \& Public Health Preparedness, 7(5), 522.

[6] Wei, S. X., Yin, A. T., \&Ma, D. P.(2014). Talented personnel problem and causes of general Practitioner system construction. Chinese Health Resources, 22(3), 227-228.

[7] Zhu, F., Liu, X. N., \&Lei, P.(2011). Research on knowledge and skills status and training Needs of community general practitioners. Chinese General Practice, 14(31), 3601-3604.

[8] Zhang, L. F., Jia, Y., \&Wu, N.(2012). The influence of comprehensive health reform on incentive mechanism of health workers and countermeasures. Chinese Journal of Health Policy, 5(9), 48-52.

[9] Jiang, H. L.(2007). Discussing the development direction of community health career from the perspective of doctor cost control: An economic analysis based on the British general practitioner system. Journal of the Postgraduate of Zhongnan University of Economics and Law, 36(6), 81-84.

[10] Li, S. J., Zhang, H. R., \&Zhu, L. N.(2015) A study on the current situation and influencing factors of general practitioners' job stress and job satisfaction. Chinese General Practice. 18(4), 387390.

[11] Fama, E. F.(1988). Agency problems and the theory of the firm. Journal of Political Economy, 88(2), 288-307.

[12] Kreps, D. M., \&Wilson, R.(1982). Sequential Equilibria. Econometrica, 50(4), 863-895.

[13] Liu, H. P., \&Zhang, S. Y.(2015). A study on the dynamic incentive model of Chinese managers based on reputation theory. Chinese Journal of Management Science. 13(4), 78-86.

[14] Kong, F., \&Zhang, W.(2014). On long-term incentive optimal combination model of state-owned enterprise managers based on double reputation. Chinese Journal of Management Science, 22(9), 133140.

[15] Shi, Q. Q., Zhu, J. B., \&Sheng, Z. H.(2017).Dynamic incentive mechanism for the suppliers of mega project considering double reputation. Journal of Systems \& Management, 26(2), 338-345. 
1

\section{Figures}

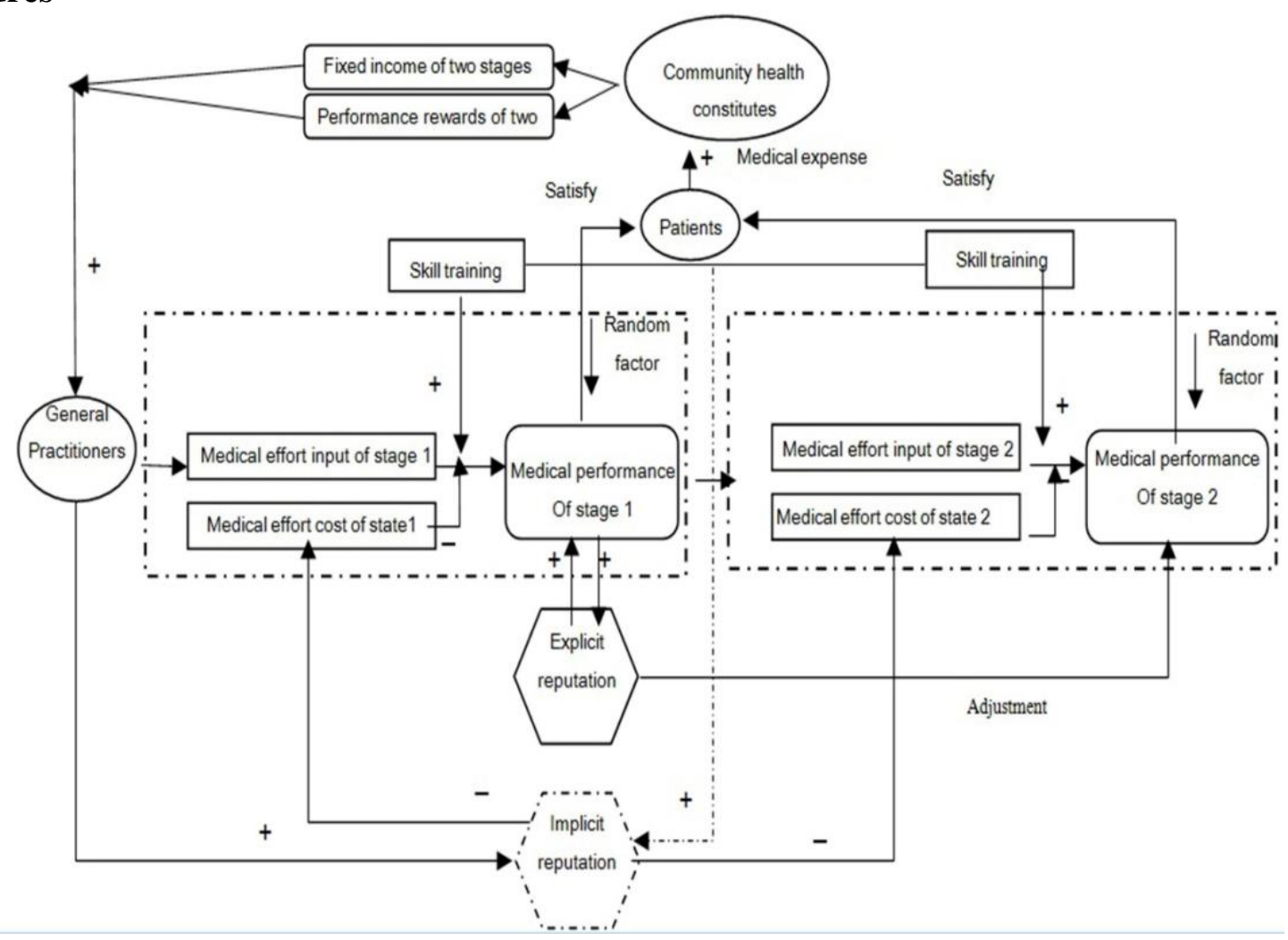

Fig. 1 GPs two-stage dynamic reputation incentive mechanism model 


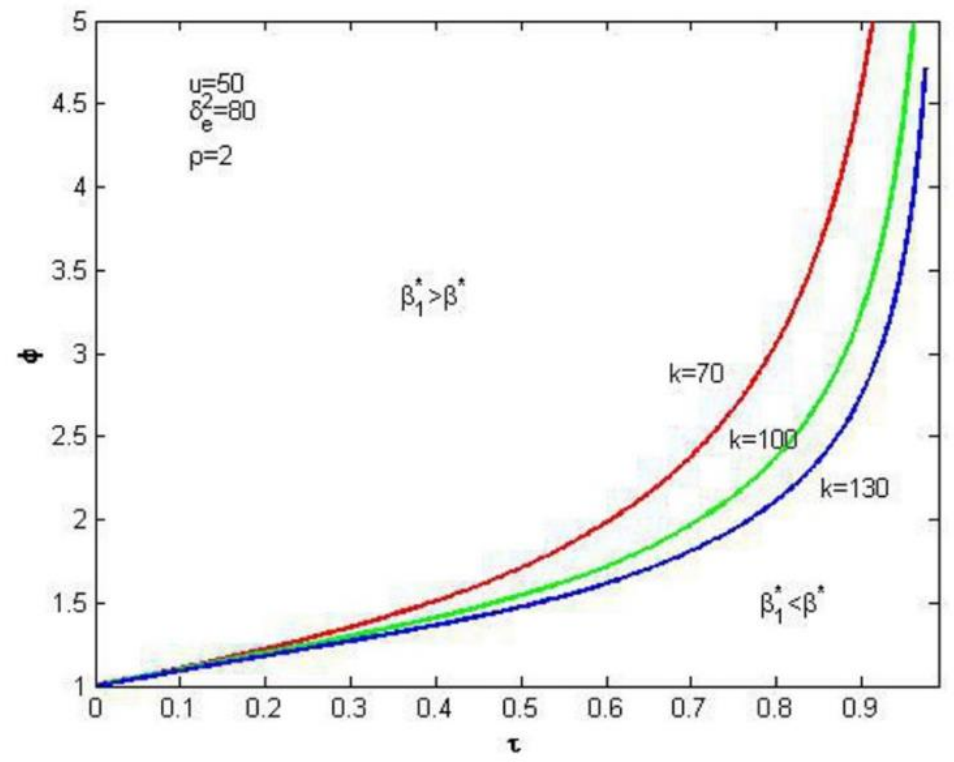

Fig. 2 The effect of $\varphi, \tau$ on the size relationship of $\beta_{1}^{*}$ and $\beta^{*}$

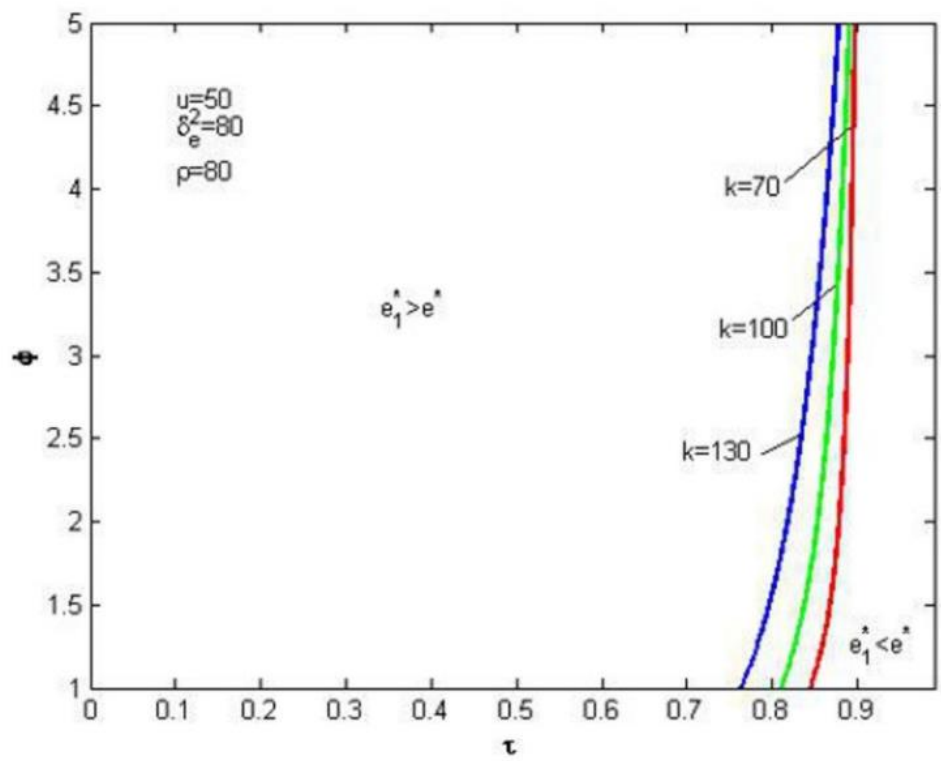

3

Fig. 3 The effect of $\varphi, \tau$ on the size relationship of $e_{1}^{*}$ and $e^{*}$ 
Figures

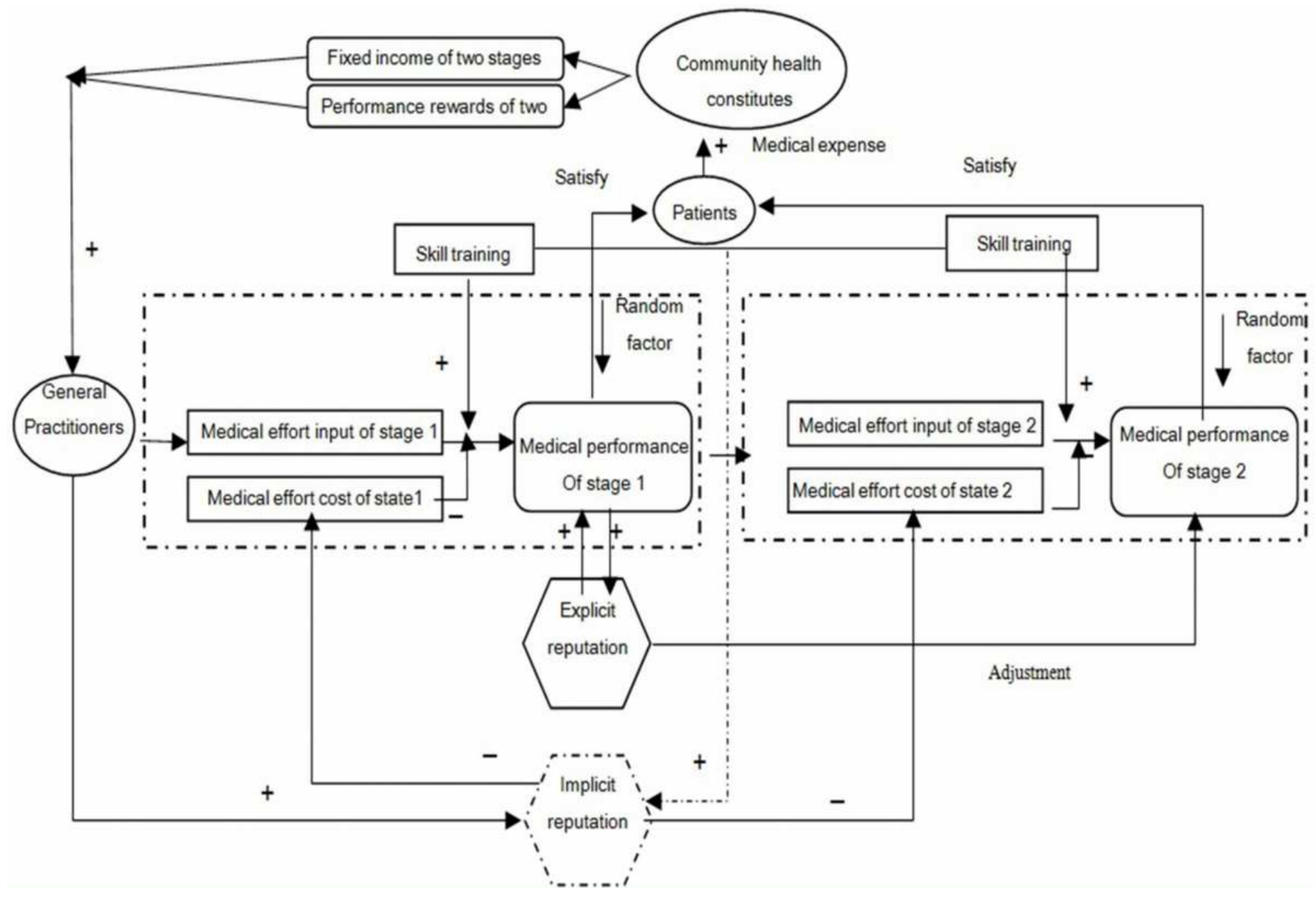

Figure 1

GPs two-stage dynamic reputation incentive mechanism model 


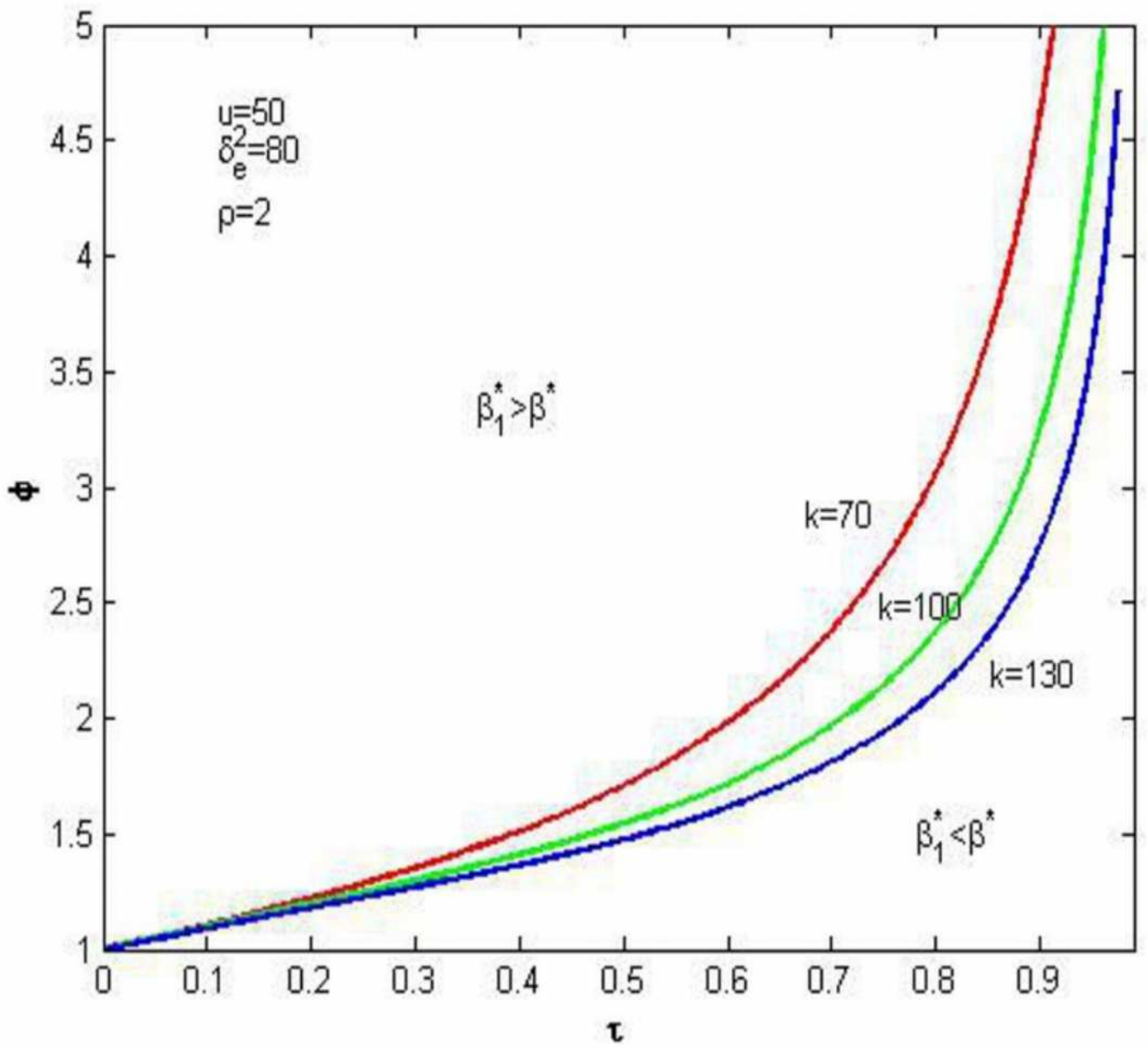

Figure 2

The effect of on the size relationship of $\beta 1^{\star}$ and $\beta^{*}$. 


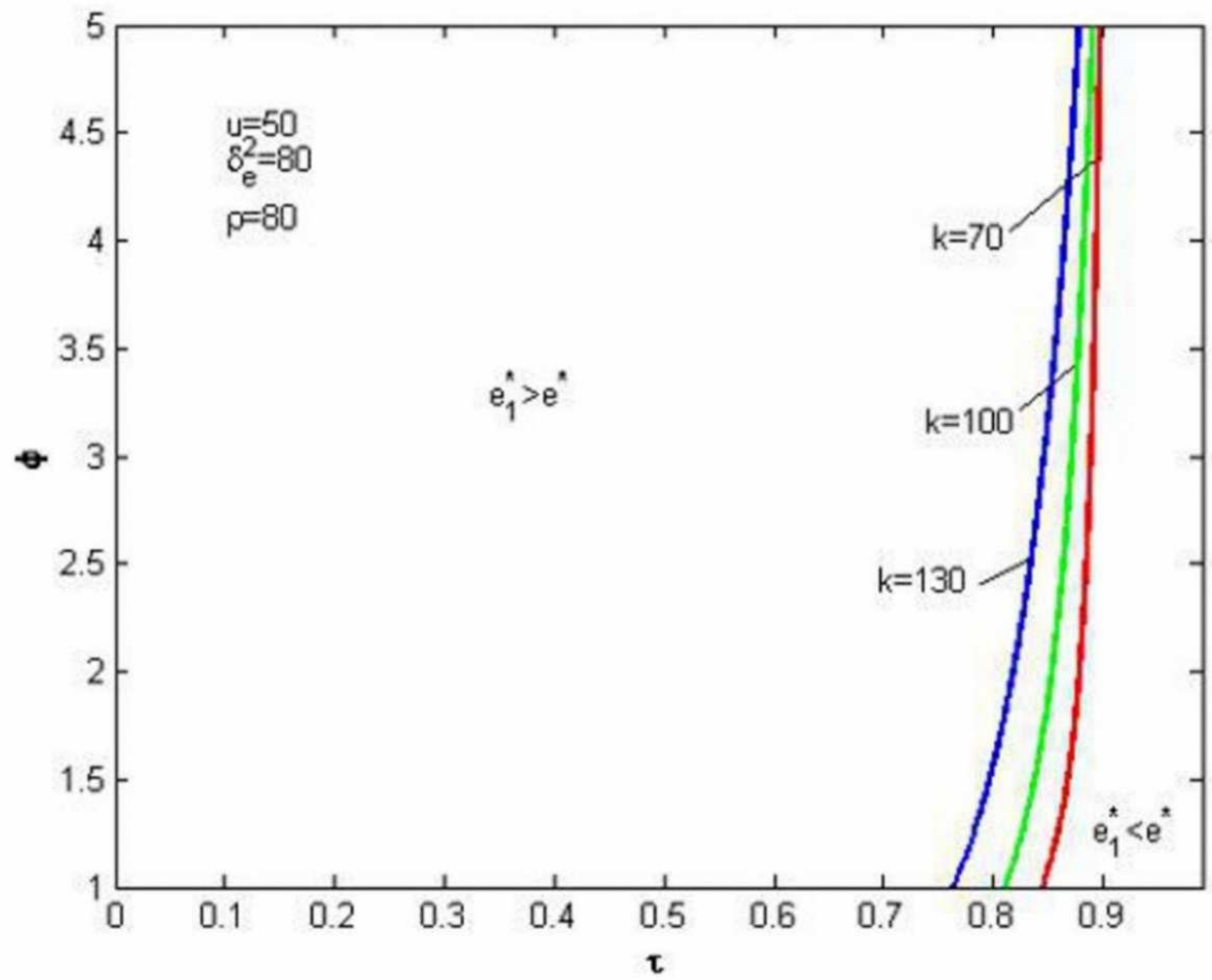

Figure 3

The effect of $\varphi, \tau$ on the size relationship of $\mathrm{e}^{\star}$ and $\mathrm{e}^{\star}$ 